\title{
Analysis on Differences between English and Chinese Sentence Structure
}

\author{
Jian Zhou \\ Wuhan Donghu University, Wuhan, Hubei, 430070
}

Keywords: English and Chinese; sentence structure; language sensitivity; locale

\begin{abstract}
English learning requires skills, and learners need to have high language sensitivity and understand the differences between English and Chinese. Especially for domestic students who are exclusively in Chinese language environment, language habits and language thinking are easily solidified, and the understanding of vocabulary and sentences is based on Chinese language, which causes many problems in English learning, that is, there isn't enough understanding of the sentence, and it is impossible to quickly master the learning skills. With the strengthening of communication at home and abroad, the importance of English learning has become more and more serious. People began to pay attention to the relevant skills of English learning and began to pay attention to the analysis of English-Chinese bilingual differences. Based on the relevant literature, this paper analyzes and discusses the differences in sentence structure in English and Chinese, and hopes to provide suggestions for English learning through the analysis of the differences between English and Chinese sentence structures.
\end{abstract}

\section{Introduction}

English learning is a long-term process that requires students to have a high level of English sensitivity and be able to clarify the differences between English and Chinese. In China, the vast majority of students are born in a Chinese environment. They are born in China, speak Chinese, and their logical thinking is completely Chinese. This leads to the students' reflective use of their own English. Chinese language logic is to think about problems and analyze problems, which leads to inefficiency in learning English. Due to the differences between Chinese and Western cultures and the different language expression habits, there is a big difference between Chinese and English in grammatical structure and sentence structure. Therefore, students will face greater difficulties in English learning and Chinese-English translation. English learning is a very deep learning. When learning English, you need to maintain the rigor of language, but you can't stick to the form, too rigid. For students, when they are studying English, students are required to correctly understand the meaning of sentences, correctly grasp the differences between Chinese and English, maintain the rigor and consistency of translation in translation, and ensure the correct conversion of Chinese-English bilingual.

\section{English hypotaxis and Chinese parataxis}

English is different from Chinese. In most English sentences, it is composed of the structure of the subject, predicate and object. When expressing, the elements of the whole sentence are complete and coherent. For most English sentence structures, the subject-predicate is the key component, and the sentences are mostly composed of conjunctions, gerunds, participles plus relational words and some infinitives. The application of these elements maintains the integrity of the entire sentence. However, Chinese sentences do not pay special attention to the overall integrity of the sentence, but pursue the completeness of the meaning expression. As long as it can express its own intention, it will not make much demands on the structure, order and integrity of the sentence. The difference in sentence structure is very obvious. For example, "lucky are only for the left hands", formerly translated as "lucky is always reserved for the prepared hands" [1]. The correct translation should be "Fortunate and always reserved for the hard work". As the famous American linguist Eugene A. Nida said in his book "Language, Culture and Translating": "There are many differences between 
English and Chinese, and there are great differences in concept, thinking, structure, etc. But from a certain point of view, the biggest difference between English and Chinese is in form and meaning." Eugene A. Nida also said in "Exploration of Translation Science" that English itself is a form of language and needs the use of linguistic formal means (including lexical means and morphological means) to achieve the connection of words or sentences. The so-called "intentional" means to achieve the connection between them by means of the meaning or logical connection of words or sentences without means of linguistic form means. Specifically, the logical relationship between English single sentences needs and must rely on logical conjunctions [2]. And this language habit of using other elements to express logical relationships has become the biggest difference between English and Chinese sentence structure. In English, when the sentence is expressed, it will rely on the conjunction to clarify the logical relationship of the sentence, such as "as well as, and so on", and other conjunctions that represent the parallel relationship, as well as "not, because, however, but, etc". The connective words of the table transition relationship, the connective words such as "as a result, so", which indicates causal relationships, the connective words such as "unless, if”, etc., are all connected words to express the logical relationship.

\section{English emphasizes bottom decoration, and Chinese emphasizes front decoration}

In English, the subject-predicate structure is the basic structure of the sentence, and the subject usually suggests the subject of the sentence. The subject of an English sentence is a person or thing. The grammar requires that the part of speech of the subject must be a noun or a noun phrase. A Chinese sentence is divided into two parts: the theme and the description part. Subject is not limited to noun. In addition to noun, verbs, adjectives, time and place conditional adverbials can be used as the subject of the sentence. In addition, the subject of a Chinese sentence is not necessarily the subject of a sentence. When the sentence is clear, the subject can be omitted. In this case, the subject of the sentence is not the subject. This is usually ignored by English learners and is one of the important manifestations of "Chinglish" [3]. For Chinese, the basic sentence structure, that is, the general sentence pattern is composed of the subject-predicate, which is the same as English, and the general sentence of English is also composed of the subject-predicate. However, for English, the lyrics may be in front of the attributive and singular sentences. It is also possible to place the qualifiers after the attributive and singular sentences. Generally speaking, if the modifier is a single word, it is usually placed in front of the sentence; and if the modifier is a clause, it should be put at the bottom the sentence. However, for Chinese, the vast majority of qualifiers are prepositions, and there are very few postpositions. This difference in sentence structure leads to the opening of many English structures in the end of the sentence, which is quite different from Chinese. As shown below, for example:

Example: He was shopping.

He was shopping at the mall.

He was shopping in a gorgeous mall.

He was shopping with his friends in a gorgeous mall.

However, in Chinese sentences, the modifiers are mostly prepositional.

I don't eat at night because I have to lose weight.

Original translation: I will not eat at night because I have to lose weight.

Translating: I don't eat at night because I want to lose weight.

\section{English sentence focuses on the front, and Chinese sentence focuses on the back}

We all know that language is the expression of thought, which determines that English and Chinese are basically the same, the basic structure is similar, and whether it is English or Chinese, there will be a center, a focus, and through other vocabulary and he superposition of sentence patterns expresses this focus clearly [4]. As Eugene A. Nida said in "Language, Culture and Translating," all the complex sentences are just to illustrate a point of view, a fact, a center, and the importance of multiple elements in a sentence is not equal. There is a focus and side focus, and the 
translator must accurately grasp this focus and express the author's ideas perfectly [5]. However, although both English and Chinese have a focus, the focus of English is generally on the front, while the focus of Chinese is basically on the back. English itself is relatively open. In terms of the expression of thought and the habit of language, it is more likely to be direct and straightforward, while Chinese sentences are more reserved and introverted, prefer a gradual language expression. As shown in the following example:

Example: (1) Bob criticized Sarah, not because Bob mad with Sarsh, but because Bob love Sarah.

Originally translated as Bob criticized Sarah not because Bob was angry with Sarah, but because Bob loved Sarah.

The correct translation should be because Bob loves Sarah to criticize her.

(2) We have maked the deal after many peoples hard work.

The original translation has reached a consensus for us, after many efforts.

It is translated as we have reached an agreement after many people's efforts.

\section{English has more compound sentences, Chinese more simple sentences}

As the author said in the first part, in most English sentences, the structure of the subject-predicate is composed. When expressing, the elements of the whole sentence are complete, coherent and complete. When performing language expression, we can clearly find that the sentence structure is mostly composed of conjunctions, gerunds, participles plus relational words and some infinitives. Through the application of these elements, the integrity of the whole sentence is maintained. Compound sentences, multiple parallel sentences, belong to the tree structure [6]. However, Chinese does not pay attention to the integrity of this structure, but uses a lot of phrases or simple sentences to form a paragraph to express the author's meaning. In Chinese language, the subject's function is weak, and the length is arbitrary. As long as the meaning can be expressed completely, there is no strict requirement for the sentence pattern.

\section{Differences in thinking pattern between English and Chinese sentence structures}

Due to the different language environments, different ways of thinking, differences in language habits and logical habits, there will be a big gap in the way of thinking between English and Chinese sentence structures. In addition to the different emphasis of the center, the difference between the front and the rear, the English and Chinese sentence structure in the use of logical conjunctions, there are many differences in the subject dynamics. For English-Chinese bilingualism, it is precisely because of different language habits that different thinking logics ultimately lead to differences in sentence structure [7]. Chinese people are subtler, language expression is more restrained, while English is more open, and the expression more focuses on structural clarity and logical rigor. In the comparison of English and Chinese sentences, an important difference in our general cognition is that the frequency of passive sentences in English is very high, while in Chinese there are few passive sentences. This difference is one of the differences between the English and Chinese ways of thinking, that is, considering the difference in perspective. Passive sentences in English are often used in styles that express objective facts or attitudes in scientific articles, academic reports, news reports, and landscape descriptions. However, Chinese expression is influenced by the Chinese subject's thinking. It is often used to indicate the actioner's behavior, use active sentences more, or express passive meaning from an active perspective.

\section{Conclusion}

Due to the differences between Chinese and Western cultures and the different language expression habits, there is a big difference between Chinese and English in grammatical structure and sentence structure. Whether in the difference between the form and the meaning, the difference between the modification and the center of gravity, or the difference between the compound 
sentence and the simple sentence, there is a clear difference between English and Chinese. And to understand the differences in sentence structure can effectively help us to learn English, master the skills of learning, and improve the efficiency of English learning. Due to the limitations of knowledge, the author's understanding of the differences between English and Chinese structure is relatively superficial and simple, and needs more correction and comments of experts and scholars.

\section{References}

[1] He Zhiying. Cultivating students' sensitivity to English and Chinese language thinking through contrast between English and Chinese vocabulary and sentence structure[J]. Journal of Inner Mongolia Normal University (Educational Science Edition), 2015, 25(01): 76-78.

[2] Zhang Tianyu. Application of translation compensation strategy in Nothing Down for the 2000s Chinese translation [D]. Liaoning Normal University, 2017.

[3] Sun Yanghai. Analysis of English and Chinese Language Differences and Common English Writing Skills Review on the Evaluation of English and Chinese Sentence Structures in "Investigation of Contemporary English Writing Teaching Theory and Practice"[J]. Contemplation Sciences, 2015(23): 85.

[4] Huang Fang. Azalea. A study on the structural changes in Chinese-English translation of Chinese and English in the textbooks of primary English and Chinese [D]. Hunan Normal University, 2018.

[5] Jingmin Li. Xinghe vs. yihe in English-Chinese translation - Developing students' differences in thinking in English and Chinese through contrastive analysis of English and Chinese lexical and syntactic structures [J]. Perspectives,2011,19(3).

[6] Xuedong SHI. Cultural Approach to English-Chinese Metaphor Translation[J]. Higher Education of Social Science, 2014, 6(3).

[7] Mary John Smith. Jerome C. Kuperman. On the Translation Strategies of Long English Sentences in Literary Works from the Perspective of Translation Stylistics [J]. Journal of Public Relations Research, 2016, 15(2). 